\title{
La estimulación temprana como base para los procesos de enseñanza-aprendizaje en la educación infantil.
}

Early stimulation as a basis for the teaching-learning processes in early childhood education.

Ruth Sayonara Ríos Bayas. ${ }^{\text {, Sandy Janeth Coral Padilla. }}{ }^{2}$, Orlando Rodrigo Carrasco Coca.

${ }^{3}$ \& Cristina Elizabeth Espinoza Regalado. ${ }^{4}$

Recibido: 22-11-2020 / Revisado: 30-11-2020 /Aceptado: 19-12-2020/ Publicado: 02-01-2021

\begin{abstract}
.
DOI: https://doi.org/10.33262/cienciadigital.v5i1.1543

Introduction. The application of Early Stimulation programs to promote comprehensive training and maximum development in children has been a priority task, however, making these the basis on which the teaching-learning processes are sustained continues to be a great challenge, among other aspects due to the diversity itself and the lack of equity when applying them. Objective. Reflect on the relationships that exist between both processes and the importance of Early Stimulation in achieving significant results in the teaching-learning process. Methodology. The study contemplated a descriptive, non-experimental methodology based on the Systematic Review of Literature (RSL), which made it possible to consult 29 sources related to the subject, identifying 11 relevant investigations under the inclusion and exclusion criteria. Results. 1. It is confirmed that we are in the presence of a process that is of great importance for the child's life, which in terms of its terminology has had different definitions, agreeing that it is aimed at preventing and / or compensating for possible anomalies, in the same A measure that seeks the comprehensive stimulation
\end{abstract}

\footnotetext{
${ }^{1}$ Universidad de las Fuerzas Armadas. (ESPE) Carrera: Educación Inicial. rsrios@espe.edu.ec ORCID ID: https://orcid.org/0000-0002-5468-1441

${ }^{2}$ Universidad de las Fuerzas Armadas. (ESPE), Carrera: Educación Inicial sjcoral@espe.edu.ec ORCID ID: https://orcid.org/0000-0001-8992-8355

${ }^{3}$ Universidad de las Fuerzas Armadas. (ESPE), Carrera: Pedagogía de la Actividad Física y Deportes orcarrasco@espe.edu.ec ORCID ID: https://orcid.org/0000-0001-6995-2534

${ }^{4}$ Institución Educativa Fiscal "Juan Genaro Jaramillo" crisespinoza86@ hotmail.com ORCID ID: https://orcid.org/0000-0002-0249-0600
} 
of all areas of the child's development, in direct connection with the socio-educational and family context. 2 . To form the basis of the teaching-learning process, it presupposes determining what the objectives are to be achieved, being preceded by the realization of multidisciplinary diagnoses and having the active participation of the child and his family environment, as well as teachers or specialists with the skills required to detect, characterize, evaluate and intervene. Conclusions. It is considered a highly significant process and of great importance for the successful development of the teachinglearning process, as long as it is carried out from the vision of the integral development of the child, contemplating the stimulation of all areas. Its value lies in conceiving this from multidisciplinary, equitable and inclusive positions.

Keywords: Early stimulation, teaching-learning process, early childhood education.

\section{Resumen.}

Introducción. La aplicación de programas de Estimulación Temprana para potenciar la formación integral y el máximo desarrollo en los niños, ha venido constituyendo una tarea prioritaria, sin embargo, hacer que estos sean la base sobre la cual se sustenten los procesos de enseñanza -aprendizaje continúa siendo un gran reto, entre otros aspectos por la propia diversidad existente y la falta de equidad al momento de aplicarlos. Objetivo. Reflexionar sobre las relaciones que se dan entre ambos procesos y la importancia que tiene la Estimulación Temprana en el logro de resultados significativos en el proceso de enseñanza-aprendizaje. Metodología. El estudio contempló una metodología descriptiva, no experimental con base en la Revisión Sistemática de la Literatura (RSL), la cual facilitó consultar 29 fuentes relacionadas con el tema, identificándose bajo los criterios de inclusión y exclusión 11 investigaciones relevantes. Resultados. 1. Se confirma que estamos en presencia de un proceso que reviste gran importancia para la vida del niño, el cual en cuanto a su terminología ha tenido diferentes definiciones, coincidiéndose en que está encaminado a prevenir y/o compensar posibles anomalías, en la misma medida que busca la estimulación integral de todas las áreas del desarrollo del niño, en vínculo directo con el contexto socioeducativo y familiar. 2. Para que constituya la base del proceso de enseñanza aprendizaje, presupone, determinar cuáles son los objetivos a alcanzar, estar precedido de la realización de diagnósticos multidisciplinares y contar con la participación activa del niño y de su entorno familiar, así como de docentes o especialistas con las competencias requeridas para detectar, caracterizar, evaluar e intervenir. Conclusiones. Se considera un proceso altamente significativo y de gran importancia para el desarrollo exitoso del proceso de enseñanza-aprendizaje, siempre y cuando se realice desde la visión del desarrollo integral del niño, contemplando la 
estimulación de todas las áreas. Su valía radica en lograr concebir este desde posicionamientos multidisciplinares, equitativos e incluyentes.

Palabras claves: Estimulación Temprana, proceso de enseñanza-aprendizaje, educación infantil.

\section{Introducción.}

El desarrollo de programas de Estimulación Temprana para dar atención a la primera infancia ha venido constituyendo una tarea prioritaria en los diferentes países. Sus inicios marcan en el decursar histórico de los fundamentos educativos un antes y un después. Múltiples son los estudios e investigaciones que han sustentado su importancia y utilidad en el proceso formativo e integral del niño. De ahí que estemos ante un tema de especial importancia y significación.

Al analizar los orígenes de los procesos de estimulación temprana, tanto a nivel internacional como nacional se puede apreciar que cada país, ha ido aproximándose desde las diferentes perspectivas a dar atención a la primera infancia, incluso desde el vientre materno; lo paradójico de esta situación es que aún no se alcance los niveles necesarios y deseados entre otros aspectos porque no se realiza de forma equitativa e inclusiva para todos los niños y niñas comprendidos en estos rangos de edades.

Dentro de todo esto un elemento fundamental radica en la relación que se da entre los objetivos del proceso de Estimulación Temprana como base para el desarrollo exitoso de los procesos de enseñanza-aprendizaje. Es por ello que el objetivo del presente estudio se centra en: Reflexionar sobre las relaciones que se dan entre ambos procesos y la importancia que tiene la Estimulación Temprana en el logro de resultados significativos en el proceso de enseñanza-aprendizaje.

\section{Generalidades de la Estimulación Temprana. Aproximación a los antecedentes,} actualidad conceptualización y objetivos.

\subsection{Una aproximación a los antecedentes y actualidad.}

Atender a los niños desde las primeras edades es además de prioritario una obligación del estado en cualquier país, independiente a que su condición pueda estar asociada o no a una discapacidad. Maqueira, (2020). Es por ello que los procesos de estimulación temprana ocupan un lugar fundamental en el sistema de acciones e influencias que reciben los niños en estas primeras edades, los cuales están diseñados para potenciar su máximo desempeño y estimular todas las áreas de su desarrollo. 
En Latinoamérica y también en Ecuador atender a los niños en la primera infancia desde hace varios años ha constituido una primacía, sin embargo, aún se está muy lejos de lograr alcanzar lo que realmente se requiere. Los análisis realizados al respecto por Barreno, \& Macías, (2015) y Escobar, et al.,(2020), enfatizan que en estos países se han estado desarrollando programas de atención a la primera infancia desde hace más de un siglo y medio, señalando que estos procesos tienen sus primeras experiencias en programas de tipo asistencial para posteriormente incursionar en el contexto educativo mediante el trabajo en Jardines Infantiles $\mathrm{u}$ otros centros similares como es en el caso de Ecuador los Centros Infantiles del Buen Vivir (CIBV).

Lo cierto es que al analizar los orígenes de los procesos de estimulación temprana, tanto a nivel internacional como nacional se puede apreciar que cada país aparte a su cultura o nivel de desarrollo ha ido aproximándose cada vez más con mayor intencionalidad a ofrecer programas de atención que permitan estimular el desarrollo infantil incluso desde el vientre materno, lo paradójico de esta situación es que debido a diferentes causas no se realiza de forma equitativa e inclusiva para todos los niños y niñas comprendidos en estos rangos de edades.

Cabe mencionar dentro de los programas más significativos desarrollados en Latinoamérica los siguientes:

- Colombia, (Estrategia de Atención Integral a la Primera Infancia "De Cero a Siempre") Argentina, (Plan Nacional de Acción por los Derechos de los Niños, Niñas y Adolescentes)

- Panamá, (Plan de Atención Integral a la Primera Infancia - PAIPI)

- Uruguay, (Plan Nacional de Desarrollo Integral de la Primera Infancia)

- Chile, (Subsistema de Protección Integral a la Infancia Chile Crece Contigo)

- México, (Estancias Infantiles)

- Brasil (Brasil Cariñoso)

- Guatemala (Políticas Públicas de Desarrollo Integral de la Primera Infancia),

- Cuba (Programa Educa a tu hijo)

- Perú (Sistema de Atención Integral al niño y adolescente),

- Nicaragua (Política Nacional de Primera Infancia “Amor para los más chiquitos y Chiquitas.

Todos estos países mediante sus diferentes programas han venido prestando atención a los niños en las primeras edades. En Ecuador la Estrategia Infancia Plena, se alinea con el Plan Nacional para el Buen Vivir 2013-2017, que plantea las políticas de la primera infancia para el desarrollo integral como una prioridad de la política pública. Dicha estrategia, articula el trabajo intersectorial de los Ministerios de Inclusión Económica y Social, Educación y Salud, los cuales cuentan con un paquete de servicios para la atención de niños de 0 a 5 años. 
En este mismo orden de ideas es bueno reconocer que en el caso de Ecuador evidentemente se han dado avances en cuanto al desarrollo de programas que permitan la atención integral del niño en los primeros 6 años de vida. La normativa legal desde la propia Constitución de la República, el Plan Nacional del Buen Vivir, la Agenda de Atención a las personas con Necesidades Educativas así lo confirma. Lo anterior ha permitido identificar donde están las mayores fortalezas como también los aspectos aun deficitarios, permitiendo reorganizar mediante los diferentes entes gubernamentales las estrategias de trabajo e intervención a seguir.

Si es consideración de los autores de la presente investigación que dada la propia diversidad existente unida a otras causas aún estos programas no satisfacen la demanda y las realidades que este tema impone en el contexto ecuatoriano; ocurriendo lo mismo en la mayoría de los países latinoamericanos, donde los altos índices de pobreza, la desnutrición infantil, la falta de equidad, de recursos y de apoyos económicos, políticos, y educativos matizan realidades y aconteceres muy difíciles para la población infantil y sus familias.

\subsection{Una aproximación a la Conceptualización y los objetivos de la Estimulación Temprana.}

Múltiples han sido las investigaciones que se han venido desarrollando a lo largo del tiempo en relación con la Estimulación Temprana, los cuales además de conceptualizar el proceso como tal, han contribuido con importantes aportes a explicar y demostrar su utilidad en estas edades.

Los autores del presente estudio sin pretender abarcar toda la variedad de definiciones, terminología y conceptualizaciones que se ha dado tanto en la literatura internacional como nacional sobre tema, intentaremos acercarnos a los principales argumentos que desde el punto de vista teórico nos permiten mediante la revisión bibliográfica realizada tener un acercamiento tanto a la conceptualización como a los objetivos de la Estimulación Temprana, sin dejar de contemplar que este término ha sido reconocido por la literatura de diferentes formas, dentro de las cuales se ha relacionado con otros como: Atención Precoz, Estimulación Precoz, Atención Temprana, Intervención Temprana. De todo ello se deduce una primera idea a nuestro criterio muy importante y es que independientemente de las diferentes terminologías empleadas es un proceso que está relacionado directamente con la necesidad de anticiparnos en el tiempo, es decir, mediante las técnicas, ejercicios y acciones empleadas se prevé la estimulación de las diferentes áreas del desarrollo para potenciar el máximo desempeño desde las primeras edades.

En esta línea de ideas los estudios precedentes indican y según la investigación realizada por Huamán, (2018), que este término tuvo sus orígenes en la Declaración de los Derechos del Niño, en 1959, definiéndose como “... una forma especializada de atención a los niños que 
nacen en condiciones de alto riesgo biológico y social, en el que se privilegia a aquellos que tienen familias marginales, carenciadas o necesitadas...”. (Huepp, 2005, p. 16)

La Federación de Enseñanza de Andalucía, (2011), al definir la Estimulación Tempana, refiere que se trata del conjunto de estrategias, actividades y métodos sistematizados, continuos y repetitivos, destinadas a potenciar el desarrollo de las capacidades motrices, tanto gruesas como finas, las capacidades cognitivas-intelectuales, socioafectivas y estética de los niños que se encuentran en las edades entre 0 y 6 años". Mientras que otros estudios lo definen como el proceso de estructuración del conjunto de acciones dirigidas a los niños, desde su nacimiento hasta los 6 años, el cual les permite el desarrollo integral de sus capacidades, dígase capacidades motrices, cognitivas, socioafectivas y del lenguaje, entre otras.

Según el Real Patronato de Prevención y de Atención a personas con discapacidad al referir el "Libro Blanco de la Atención Temprana" del año (2000), define por Atención Temprana, al conjunto de intervenciones dirigidas a la población infantil de 0-6 años, a la familia y al entorno, que tienen por objetivo dar respuesta lo más pronto posible a las necesidades transitorias o permanentes que presentan los niños con trastornos en su desarrollo o que tienen riesgo de padecerlos. Considerando en ello todas las intervenciones, que deben comprenden la globalidad del niño, las cuales deben ser planificadas por un equipo de profesionales de orientación interdisciplinar o transdisciplinar".

Por su parte la UNICEF en el (2011), indica que se trata de conjunto de acciones tendientes a proporcionar al niño las experiencias que éste necesita desde su nacimiento, para desarrollar al máximo su potencial psicológico, logrando, un cierto grado de interés y actividad, condición necesaria para lograr una relación dinámica con su medio ambiente y aprendizaje afectivo". Apunta que ayuda a fortalecer el cuerpo y a desarrollar las emociones y la inteligencia del niño, integrando en este proceso actividades a su juego diario

Ordoñez, y Tinajero, (2012), definen la estimulación temprana y estimulación adecuada, como una teoría basada en las neurociencias, en la pedagogía y en la psicología cognitiva y evolutiva, que se implementa mediante programas fundados con la finalidad de ayudar al desarrollo integral del niño. Mientras que Terré (2012), plantea que la estimulación temprana es el cómo el conjunto de medios, técnicas y actividades que se aplican en niños y niñas con el fin de desarrollar al máximo sus capacidades cognitivas, físicas y psíquicas el, señala el autor que se puede entender como el conjunto de actividades y rutinas diarias favorecedoras del desarrollo integral y armónico de las capacidades y/o potencialidades del bebé.

En el contexto ecuatoriano las investigadoras Barreno, \& Macías, (2015), reafirman que la estimulación temprana mejora las condiciones físicas, emocionales, cognitivas y sociales de los niños y niñas, potenciando la psicomotricidad, para elevar el rendimiento escolar, y los 
resultados de evaluación en todas las etapas educativas. (pp 111); precisamente porque como bien señalan en su investigación las autoras precedentes la metodología de la estimulación está dirigida a varias dimensiones dentro de las cuales se encuentran: la socioafectiva, corporal, cognitiva, comunicativa, estética y ética y valores; es decir se da en función de promover el máximo desarrollo del niño en la edad inicial.

Para Esteves, et al., (2018), la estimulación temprana o también llamada atención temprana consiste en proporcionar al bebe un mayor desarrollo tan físico como intelectual y social para que de esta manera sus habilidades y capacidades le permitan ser mejor de lo que hubiera sido estimulándose de manera natural, esta estimulación incluye un conjunto de actividades que pueden ser aplicadas desde que nace hasta los 6 o7 años del niño, franja de edad con mayor plasticidad cerebral.

Por su parte Caamaño, (2020), lo define como un proceso basado en la repetición de ciertos eventos sensoriales en la relación diaria con él bebe quien, mediante este proceso, establece un mayor control respecto a sus propias emociones, elevando su seguridad e iniciativa, mientras que paralelamente sentirá enorme satisfacción al descubrir que es capaz de realizar las cosas por sí mismo, provocando una ampliación de su habilidad mental, facilitándole el aprendizaje, haciéndoles desarrollar destrezas para su auto estimulación, mediante el juego libre y del ejercicio de la curiosidad, la exploración y la imaginación.

En relación con ello, interviene de igual manera, el aspecto relacionado con la edad conveniente para iniciar la estimulación temprana y hasta cuando se realiza la misma, al respecto, de igual forma existen diversos criterios, entre los que podemos citar a Carreño y Calle (2020), quienes son de la opinión que "referente a la edad para que el niño reciba la estimulación temprana, recomiendan comenzar incluso desde antes de su nacimiento, con la aplicación de música y mimos afectuosos, y hasta los 6 años. Expresan también que existen programas diseñados especialmente para los recién nacidos hasta los 3 años, fundamentados precisamente, en que es en los primeros 36 primeros meses cuando es mayor la plasticidad cerebral y, por ende, el beneficio de los estímulos puede ser mayor".

Se concuerda con Maqueira, (2020) en que se trata de un conjunto de acciones científicamente organizadas y estructuradas oportunamente, encaminadas a ofrecer diferentes estímulos y niveles de ayudas individuales y diversos, desde antes del nacimiento para prevenir posibles anomalías y desarrollar el máximo de potencialidades en el niño y su entorno, de modo tal que asegure el desarrollo integral de su personalidad desde la primera infancia y para toda la vida.

Explica esta autora que se trata de un proceso de intervención altamente importante ya que permite estimular los procesos psíquicos y el desarrollo integral del niño mediante la 
realización de diversas actividades que involucran simultáneamente las esferas: intelectual, bio - psicomotora y socio-emocional, favoreciendo el desarrollo armónico de su personalidad y el establecimiento de relaciones afectivas y sociales positivas.

Otros de los conceptos dados sobre este tema y que a criterio de los autores debe considerarse, es el publicado en el sitio web de la revista UNIR (2020), el cual marca que la Educación o Estimulación Temprana como un conjunto de técnicas de intervención educativas que pretende impulsar el desarrollo cognitivo, social y emocional del niño durante la etapa infantil (de 0 a 6 años). Señalando que su metodología didáctica se asienta en dos principios básicos: Estimular y potenciar las capacidades y destrezas que muestra el niño y Compensar o prevenir cualquier déficit en su neurodesarrollo (www.unir.net)

$\mathrm{Al}$ resumir estas ideas es importante acentuar que si bien estamos en presencia de un proceso que en cuanto a su terminología ha tenido diferentes definiciones se debe destacar que en todos los casos se concuerda que se trata de ese conjunto de técnicas, acciones, actividades u ejercicios que deben estar científicamente organizados y planificados encaminados a prevenir y/o compensar posibles anomalías, en la misma medida que busca la estimulación integral de todas las áreas del desarrollo del niño y de su entorno.

Dando continuidad al análisis de las teorías precursoras sobre este tema resulta trascendental abordar los objetivos de la estimulación Temprana. De acuerdo con Barreno \& Macias (2015), el objetivo de la estimulación temprana consiste en cambiar la estimulación temprana en una práctica agradable que vaya propiciando cada vez más la relación madre-hijo, desarrollando habilidades y destrezas con calidad y calidez a través de las experiencias vividas basadas en actividades lúdicas sustentados en el progreso integral (pp 111);

En este orden es importante conocer la revisión bibliográfica ha permitido identificar que los objetivos de la estimulación temprana se refieren a los aspectos específicos sobre los que se interviene y actúa en ese proceso, así como a las condicionantes que hacen posible que ese proceso sea efectivo y adecuado.

Se ha constatado que la estimulación no solo es apropiada para niños "normales", también es de gran utilidad para mejorar de manera significativa las capacidades de los niños con necesidades de educación especial asociadas a discapacidades.

De manera general los autores coinciden con Chavarría González, (1982) y Carreño y Calle (2020), en que la Estimulación Temprana cuenta entre sus principales objetivos con los siguientes:

- La optimización del desarrollo del niño en lo relativo a sus habilidades motrices, del lenguaje, cognitivas y adaptativas.

- La prevención de déficits asociados a un riesgo biológico, psicológico o social. 
- La atención de las necesidades de la familia del niño.

- Disminuir los efectos que pudiera ocasionar una discapacidad en los niños.

Es primordial conocer que la finalidad de la Estimulación Temprana no es apresurar propiamente el desarrollo motriz y cognitivo del niño, obligándolo a vencer etapas, sino hacerle ver las posibilidades con que cuenta, creándoles situaciones de desafíos que los motive a encontrar respuestas adecuadas que contribuya en cada etapa de su desarrollo.

Al respecto coincidimos con Barrera, Flor, Flor (2018), al plantear que "el objetivo de la Estimulación Temprana, no es acelerar el desarrollo, forzando al niño a lograr metas para las que no está preparado o para cumplir, si no el reconocer y motivar el potencial de cada niño en particular y presentarle retos y actividades adecuadas que fortalezcan su desarrollo físico y psicológico".

Con la finalidad de favorecer el óptimo desarrollo del niño, las actividades de Estimulación Temprana se enfocan en cuatro áreas específicas: área cognitiva, motriz, del lenguaje y socioemocional.

El Área motriz, está referida a la habilidad que posee el niño, de moverse, desplazarse y coordinar los movimientos entre lo que observan y lo que palpan permitiéndoles agarrar objetos con las manos, dibujar y otras acciones.

El Área cognitiva, es la referida al uso por el niño, del pensamiento e interacción directa con los objetos del ambiente que le rodea, permitiéndoles comprender y relacionarse y adaptarse a las diferentes situaciones que se presentan, para ello requieren de vivencias continuas que les propicie desarrollar los niveles de pensamiento, su capacidad de razonamiento, prestar atención, cumplir instrucciones y reaccionar de manera rápida.

El Área del lenguaje, se relaciona con la habilidad de comunicarse con su entorno. La misma abarca la capacidad comprensiva, expresiva y gestual. Es primordial hablarles a los niños de manera constante para que relacione la actividad que realiza y designe los objetos que manipulan, lo que le permitirá reconocer los sonidos o palabras que intentará imitar.

El Área socio-emocional, se vincula con las vivencias afectivas que ha tenido el niño y a la socialización, permitiéndole sentirse seguro y amado al relacionarse con los demás. Mucha importancia reviste la participación de sus padres, quienes les enseñan valores y reglas de la familia y la sociedad.

Otro aspecto que se debe tener en cuenta es el relacionado con psicomotricidad, referido a la vinculación directa entre la mente y el cuerpo. La psicomotricidad indica la capacidad del niño de poder tener el control de su propio cuerpo, ya sea, al control de piernas, brazos, cabeza y tronco (motricidad gruesa) o al control de manos y dedos (motricidad fina). Se debe 
reconocer que tanto la motricidad gruesa como fina, se desarrollan en orden progresivo, acorde a las etapas de desarrollo del niño. La evolución de la motricidad fina es fundamental para que el niño pueda intercambiar con el entorno e influye de manera determinante en el desarrollo de la inteligencia.

En este particular especial importancia tiene el estudio realizado por Sailema et al.,(2019) al explicar que el desarrollo y reconocimiento del esquema corporal implica la activación permanente del caudal de información, conocimientos y exploraciones que realiza el niño desde edades tempranas. Insistiendo en que en el caso de los niños con necesidades educativas asociadas a discapacidad es necesario replantear los procesos de intervención sobre la base procederes didácticos metodológicos que estimulen todo el desempeño del niño.

En relación con el lenguaje, el desarrollo del mismo mediante la estimulación temprana incluye las áreas:

- Lenguaje receptivo. - A través de ello, permite comprender el lenguaje y adquirir el significado de las palabras. Es decir, es la interpretación adecuada del mensaje.

- Lenguaje expresivo. - Mediante el mismo, permite al niño y a la niña expresarse por medio de gestos, señas o palabras.

- Lenguaje articulado. - Por medio de esta habilidad el niño puede emitir sonidos, combinarlos y formar sílabas, palabras, frases y oraciones que expresen ideas.

El desarrollo del lenguaje en el niño se realiza de manera continuada, al respecto (Edupeques, 2014) indica que el lenguaje en los niños se concibe como un proceso de aprendizaje socio fisiológico único e indivisible.

Derivado de todo lo anterior se asume por parte de los autores de la investigación que se presenta, la segunda idea la cual está relacionada con los objetivos de la estimulación temprana. Se ha logrado sistematizar que los objetivos son amplios; los mismos deben determinarse acorde con las características e individualidades de los niños y de su entorno, bajo los principios del diagnóstico oportuno basado en las potencialidades y en la prevención temprana de posibles desajustes, considerando siempre la estimulación y potenciación de todas las áreas del desarrollo.

\subsection{La Estimulación Temprana como base para el proceso de enseñanza- aprendizaje en la educación infantil.}

La educación en edades tempranas y especialmente en los primeros 6 años de vida de los niños, constituye de por sí un reto, dado que la misma por su importancia, se presentan al mismo nivel, que la satisfacción de sus necesidades básicas, tales como nutrición y salud. La 
literatura consulta enfatiza en la estrecha relación que se da entre las experticias y estímulos que recibe el niño y el nivel de destrezas, habilidades y conocimientos que adquiere. De ahí que resulte fundamental propiciar ambientes favorables y estimulantes.

Diversos autores se han referido al tema, entre los que podemos citar a Ramírez, Patiño y Gamboa (2014), quienes se pronunciaron indicando que la educación de los niños en los primeros años de vida constituye un desafío, por lo que requiere del diseño de propuestas de trabajo pedagógicos que les proporcione posibilidades de enseñanza y aprendizaje consecuente con sus necesidades e intereses. Insisten en la necesidad de proporcionarles herramientas de alta calidad afectiva y cognitiva que les permita el pleno desarrollo de sus capacidades y considere sus limitaciones.

Estos mismos autores remarcaron la importancia que tiene, que el docente incluya en sus propuestas pedagógicas, los contextos sociales en que se desenvuelve el niño, del cual uno de los ambientes fundamentales es la familia, dado que constituye un eslabón indispensable en el proceso de desarrollo y aprendizaje de los niños. En este sentido gran particularidad reviste la etapa inicial de escolaridad, cuando el niño enfrenta otro medio social diferente al familiar, convirtiéndose en un paso superior en su proceso evolutivo, interactuando de diferentes maneras con el nuevo medio, donde en función del grado de actividades que realice será el desarrollo de sus capacidades.

En relación a ello, autores como Caputo y Gamallo (2010), han expresado que el nivel inicial representa la primera etapa básica para el ingreso de información del niño de un medio social diferente al familiar. En este momento de su evolución, interactúa con el medio de manera muy diversas, y mientras más variada y mayor cantidad de actividad tenga, más se desarrollarán sus capacidades, las que estarán determinadas fundamentalmente por dos importantes características, su plasticidad neuronal y el desarrollo de sus órganos sensoriales.

De igual manera ligado a ello, autores como Zapata y Ceballos (2010), han afirmado que la educación en la primera infancia, no puede basarse en actividades mecánicas y repetitivas sin sentido y poco significativos con escasos niveles motivacionales, sino que debe verse desde una perspectiva más amplia, con actividades que enriquezcan la cotidianidad de los niños, que direccione su desarrollo como un ser humano, promoviendo su libre expresión y construcción de aprendizajes.

En esta dirección, trascendental importancia reviste el aspecto propiamente del aprendizaje del niño, en el cual existen varios criterios entre los que se encuentran, el concerniente con el aspecto curricular, sobre que necesita aprender un niño y lo que está recogido dentro del mismo; el relacionado con la socialización, el juego, el desarrollo corporal y motor y el lenguaje y por supuesto lo tocante con la significatividad propia del aprendizaje. Estos conceptos han sido evaluados por varios autores, entre los que podemos citar a Zapata y Restrepo (2013), quienes expresan que los lineamientos curriculares de la Pedagogía 
Contemporánea consideran al niño como el centro del proceso educativo, en el cual los educadores, la familia y otros, son sujetos que median dentro del proceso.

En este mismo orden es trascendente tener en cuenta los aportes de Sánchez, (2011), al aseverar que en la práctica temprana es importante lograr que en el niño se estimulen y se tengan en cuenta todos los sentidos, ya que estos constituyen una vía de entrada importante para los aprendizajes; considerándose en estos los sentidos visuales, auditivos, táctiles, gustativos y olfativos, los cuales permiten que toda la gama de estimulación y experiencias llegue a las neuronas y descargue de ellas pequeñas dosis de carga positiva con una mejor calidad. Al respecto Carbajo, (2014), Albornoz \& Guzmán (2016), Agudelo, Pulgarín \& Tabares (2017), enfatizan los beneficios de la Pedagogía Montessori en los procesos de estimulación multisensorial en las primeras edades.

Otro elemento a considerar y de mucha importancia por su relación con los procesos de estimulación temprana como base para el aprendizaje en las primeras edades, es la realización del proceso de diagnóstico. En ese sentido apunta Maqueira (2020) que deberá efectuarse oportunamente, facilitando la integración de todos los componentes que rodean al niño, de modo tal que permita detectar, describir y caracterizar en qué zona de desarrollo y nivel de potencialidades o debilidades se encuentran el niño y su relación con el entorno. A juicio de Maqueira y al nuestro cada caso previo al desarrollo de los procesos de intervención o estimulación deberán ser diagnosticado de forma multidisciplinar, explorando siempre las zonas conservadas, desarrolladas y potenciadas Ello implica:

- Determinar el nivel de desarrollo del niño.

- Identificar los factores que retardan o interfieren en su desarrollo.

- Caracterización de las necesidades y potencialidades de los niños, su familia y el entorno.

- Determinación el programa de intervención en correspondencia con la valoración y los logros a alcanzar según características individuales y del entorno

- Reevaluación permanente.

Lo explicado permitirá reforzar los procesos de intervención, fomentando el aprendizaje significativo y desarrollador desde las primeras edades, además del completamiento y alimentación permanente de la estrategia de intervención a seguir en función del desarrollo de una personalidad integral, según las capacidades de aprendizaje de cada niño

Todo lo expuesto nos hace coincidir con los autores precedentes en relación a cinco aspectos esenciales dentro del proceso de estimulación temprana como base para el desarrollo del proceso de enseñanza -aprendizaje en la educación infantil. 
- La educación, enseñanza y aprendizaje en edades tempranas y especialmente en los primeros 6 años de vida de los niños, constituye un gran reto, requiriendo de atención temprana, sistemática, así como de ambientes de aprendizajes que resulten altamente motivantes y significativos para la vida del niño y su familia.

- Requiere de procesos de diagnósticos multidisciplinares que favorezcan la participación activa de todos los especialistas, del niño y de su entorno familiar como vía primaria para poder determinar correctamente sobre la base de las potencialidades o limitaciones existentes la estrategia de intervención a seguir.

- Debe además definir previamente los objetivos a alcanzar tanto desde el punto de vista curricular como socioeducativo. Es importante determinar qué necesita aprender y dominar el niño según el currículo de educación infantil y las etapas y fases del desarrollo, con énfasis en todo lo relacionado con la socialización, el juego, el desarrollo corporal, motor y el lenguaje y por supuesto lo tocante con la significatividad propia del aprendizaje cognitivo.

- Dada las propias características del cerebro infantil y la plasticidad cerebral que se da como consecuencias de los múltiples estímulos que recibe la enseñanzaaprendizaje de por si constituye un eslabón fundamental en estas edades, de ahí que resulte fundamental el trabajo integrado con la familia y todo el entorno que rodea el niño.

- El docente debe desarrollar competencias y habilidades que le permitan detectar, caracterizar, evaluar e intervenir de conjunto con el resto del personal que se encarga del desarrollo de los procesos de estimulación temprana y de enseñanza-aprendizaje en la educación infantil.

\section{Metodologia.}

Se utilizó una metodología descriptiva, no experimental con el empleo de métodos teóricos, dentro de los cuales se encuentran: el histórico-lógico, el analítico - sintético, inductivo deductivo y la revisión documental; permitiendo así realizar la RSL (Revisión Sistemática de Literatura), de conformidad con las etapas de planificación, análisis y valoración de resultados. La revisión realizada comprendió un rango de tiempo entre el 2010 y el 2020, analizándose investigaciones teóricas y experimentales publicadas en bases: tales como Scopus, PubMed, Google Académico, Scielo, Redalcy, Dialnet, Latindex, Web of Science, Publice, incluyendo la valoración de resultados de trabajos de tesis y de los aportes de organismos como la UNICEF, la Federación de Enseñanza de Andalucía, entre otros. Todos los cuales están relacionados con el tema objeto de estudio.

La selección de información y fuentes relevantes se determinó mediante los criterios de inclusión y exclusión previamente establecidos, los cuales facilitaron el control de la calidad y el rigor del trabajo presentado. 
Criterios de inclusión

1. Tipo de estudios: Estudios de revisiones teóricas o experimentales que sistematicen o desarrollen teorías en relación con la estimulación temprana y su incidencia en los procesos de enseñanza -aprendizaje.

2. Tipo de participantes: Investigadores, Docentes, Especialista y estudiantes relacionados o participantes de procesos de estimulación temprana

3. Tipo de resultados: Metodologías, modelos, programas que contemplen resultados en relación con el tema objeto de estudio

4. Tesis de Grado realizadas en los últimos 10 años en Universidades Internacionales o Nacionales sobre el tema de investigación.

Criterios de exclusión

1. Estudios o investigaciones realizadas en un rango de tiempo mayor al contemplado en la investigación.

2. Artículo en idioma diferente del español o inglés.

3. Otros estudios que por su tema o resultados no aporten elementos reveladores para nuestra investigación.

\section{Resultados.}

La revisión de la literatura precedente contempló la consulta de 29 fuentes, identificándose 11 investigaciones relevantes que contribuyeron a sistematizar las teorías relacionadas con la incidencia de los objetivos e importancia de la estimulación temprana como base del proceso de enseñanza- aprendizaje.

Al respecto se logra sistematizar y fundamentar la utilidad de la estimulación temprana en las primeras edades. El análisis realizado permitió tener una aproximación a los antecedentes y actualidad del proceso de Estimulación Temprana en el contexto Latinoamericano y también en Ecuador. Enfatizándose en la idea de que si bien es cierto que los diferentes países a lo largo del tiempo se han ido sumando al desarrollo de estos programas de forma favorable, aun no se logran alcanzar los objetivos deseados, entre otros aspectos porque independientemente de que la atención a la primera infancia es prioritaria y el proceso de estimulación temprana gira alrededor del niño y sus fundamentos parten de descubrimientos científicos sólidos que remarcan la importancia de la estimulación y atención integral a la primera infancia, este proceso no resulta equitativo e incluyente para todos los niñas y niños en los diferentes países. Denotando la necesidad de realizar acciones conducentes a su ampliación a todos los sectores sociales. 
Otro de los elementos analizados estuvo relacionado con los objetivos que contempla el proceso de Estimulación Temprana. Resultando muy significativo para nuestro estudio los aportes de Chavarría González, (1982) y Carreño y Calle (2020), los cuales señalan que en la Estimulación Temprana deben primar los objetivos relacionados con la optimización del desarrollo del niño en las diferentes áreas y habilidades: motrices, del lenguaje, cognitivas y adaptativas, así como todo lo concerniente con la prevención de déficits por diferentes riesgos y la atención integral de la familia. En este mismo orden Barreno \& Macías (2015), indican que el objetivo de la estimulación temprana debe dirigirse a la realización de prácticas agradables y placenteras encaminadas a fortalecer la relación madre-hijo, en la misma medida que permiten ir desarrollando habilidades y destrezas basadas en actividades lúdicas significativas que favorezcan el progreso integral del niño.

En correspondencia con lo anteriormente explicado el estudio sistematiza los posicionamientos teóricos que dan sustento al proceso de Estimulación Temprana como base para el desarrollo del proceso de enseñanza-aprendizaje en las primeras edades. Destacando en ello los aportes de Caputo y Gamallo (2010), Zapata y Ceballos (2010), Sánchez, (2011), Zapata y Restrepo (2013), Ramírez, Patiño y Gamboa (2014), Carbajo, (2014), Albornoz \& Guzmán (2016), Agudelo, Pulgarín \& Tabares (2017) y Maqueira (2020), los cuales confirman la utilidad de la Estimulación Temprana en los procesos de Enseñanza Aprendizaje, desde la concepción y posicionamiento científico de que mientras más rico y estimulante resulte el medio donde se desarrolla el niño mejor será su proceso de adaptabilidad a las nuevas exigencias escolares. Un niño estimulado tempranamente será un niño que independiente a su condición podrá acceder de mejor forma al aprendizaje de aspectos más complejos y profundos, dada la propia plasticidad cerebral que se da en esta etapa de la vida y el nivel de experiencia e intercambio que se ha logrado.

Derivado del análisis realizado se obtienen los siguientes resultados:

1. Al resumir estas ideas y en correspondencia con los estudios de Terre, (2012), Ordoñez, y Tinajero, (2012), Ramírez, Patiño y Gamboa (2014), Barreno \& Macías (2015), Huamán, (2018), seguidos por Maqueira (2020), entre otros, es importante acentuar que si bien estamos en presencia de un proceso que reviste gran importancia para la vida del niño, en cuanto a su terminología ha tenido diferentes definiciones, sí se debe destacar que en todos los casos se concuerda que se trata de un conjunto de técnicas, acciones, actividades u ejercicios que deben estar científicamente organizados y planificados encaminados a prevenir y/o compensar posibles anomalías, en la misma medida que busca la estimulación integral de todas las áreas del desarrollo del niño en vínculo directo con el contexto socioeducativo y familiar en el cual se desarrolla. 
2. Los avances logrados en este sentido en los diferentes países marcan un antes y un después, existiendo resultados alentadores en cuanto a las vías y programas que se desarrollan, pero paradójicamente continúa siendo un proceso en el cual por diferentes causas no es del todo equitativo e incluyente.

3. Es una etapa fundamental, caracterizada por la capacidad de adaptación del sistema nervioso y del cerebro es un factor determinante para el desarrollo posterior, se debe posibilitar que las primeras experiencias del niño con el mundo exterior, garanticen el máximo desarrollo integral de sus capacidades en vínculo directo con su entorno sociofamiliar.

4. Otro elemento a considerar y de mucha importancia por su relación con los procesos de estimulación temprana como base para el aprendizaje en las primeras edades es la realización del proceso de diagnóstico. En ese sentido se concuerda con Maqueira (2020) al señalar que este proceso debe efectuarse oportunamente, facilitando la integración de todos los componentes que rodean al niño, de modo tal que permita detectar, describir y caracterizar donde se encuentran las potencialidades o debilidades de cada caso previo al desarrollo de los procesos de intervención o estimulación. Ello implica: Determinar el nivel de desarrollo del niño, identificar los factores que retardan o aceleran el desarrollo, caracterizar las necesidades y potencialidades de los niños, su familia y el entorno, determinar el programa de intervención en correspondencia con la valoración y los logros a alcanzar según características individuales y del entorno y las exigencias del currículo de Educación Infantil.

5. Para que el proceso de Estimulación Temprana sea realmente satisfactorio y constituya la base del proceso de enseñanza aprendizaje debemos comprender que debido a la gran diversidad de los posibles usuarios en la etapa comprendida como Educación Infantil, estamos ante un gran reto, ello presupone determinar previamente cuáles son los objetivos a alcanzar, tanto desde el punto de vista curricular como socioeducativo, identificando en qué fase o etapa del desarrollo se encuentran los casos a tratar, qué conocen y qué necesitan conocer, debe además estar precedido de la realización de procesos de diagnósticos multidisciplinares, que favorezcan la participación activa de todos los especialistas, del niño y de su entorno familiar y por supuesto contar con un equipo multidisciplinar donde el docente o especialista debe poseer las competencias requeridas para detectar, caracterizar, evaluar e intervenir de conjunto con el resto del personal que se encarga del desarrollo de los procesos de estimulación temprana y de enseñanza-aprendizaje. 


\section{Conclusiones.}

- La Estimulación Temprana se considera un proceso altamente significativo y de gran importancia para el desarrollo exitoso del proceso de enseñanza-aprendizaje, siempre y cuando se realice desde la visión del desarrollo integral del niño, el cual debe contemplar la potenciación y estimulación de todas las áreas.

- La valía de todo programa de Estimulación Temprana se logra al concebir este desde posicionamientos multidisciplinares, equitativos e incluyentes.

\section{Referencias bibliográficas.}

Agudelo, L., Pulgarin, L., \& Tabares, C (2017) La estimulación sensorial en el desarrollo cognitivo de la primera infancia. Revista Fuentes https://revistascientificas.us.es/index.php/fuentes/article/download/3011/3340

Albornoz, E., \& Guzman, M. (2016) desarrollo cognitivo mediante estimulación en niños de 3 años. Centro de Desarrollo Infantil Nuevos Horizontes. Quito. Ecuador. Revista $\begin{array}{lllllll}\text { Universidad } & y & \text { sociedad. } & \text { Vol } & 8 & \text { No } & 4\end{array}$ http://scielo.sld.cu/scielo.php?script=sci_arttext\&pid=S2218-36202016000400025

Barreno, S., \& Macías, J., Estimulación temprana para potenciar la inteligencia psicomotriz (2015). Revista Ciencia UNEMI. Vol. 8 - No 15 pp. 110 - 118

Barrera H, Flor A, \& Flor F (2018)- Estimulación temprana y desarrollo psicomotor en niños de 4 a 5 años. Revista Ciencia Digital. Vol. 2, $\mathrm{N}^{\circ} 1$, p. 8-18, enero - marzo, 2018. www.cienciadigital.org.

Caamaño, B. (2020). Diversidad inclusiva. Obtenido de diversidad inclusiva: http://www.diversidadinclusiva.com/- antecedentes-históricos-de-la-estimulacióntemprana. 20 de septiembre 2020.

Caputo, M. \& Gamallo, G. (2010). Infancia, desarrollo y conocimiento: los niños y niñas y su socialización. Revista Latinoamericana de Ciencias Sociales, Niñez y Juventud. 8 (2), pp. 849-860.

Carbajo, M. (2014). La sala de estimulación multisensorial. Universidad de Valladolid. https://dialnet.unirioja.es/descarga/articulo/5084331.pdf

Carreño M, Calle A, (2020)- Aspectos fundamentales de los programas de estimulación temprana y sus efectos en el desarrollo de los niños de 0 a 6 años- Revista Científica Mundo de la Investigación y del Conocimiento (RECIMUNDO); Editorial Saberes del Conocimiento, 2020- DOI:10.26820/recimundo/Vol 4. (No1). enero.2020.499-520ISSN: 2588-073X- URL: http://recimundo.com/index.php/es/article/view/901. 
Chavarría González, M. (1982). La estimulación temprana: Apuntes sobre sus fundamentos teóricos, bases empíricas y raíces sociohistóricas: elementos para una discusión. Revista de Ciencias Sociales, 41-56.

Edupeques. (22 de octubre de 2014). Edupeques. Recuperado el septiembre de 2020, de Estimulación del lenguaje: actividades de lenguaje para niños: http://www.edupeques.com/escuela-de-padres/estimulacion-del-lenguaje-niños.html

Escobar, S., Mindiola, C., Reyes, G., Sandoval, ML., Toledo, B., Vásquez, K., (2020), Fundamentos legales, experiencias investigativas y acciones desarrolladas en América Latina y en Ecuador. Maestría en Educación Inclusiva y Atención a la Diversidad. Módulo: La estimulación Temprana en Instituciones Inclusivas. Cohorte VI. Trabajo Investigativo (sin publicar)

Esteves, ZI., Mendoza, JA., Quiñonez, M (2018), La Estimulación temprana en el desarrollo de las habilidades y capacidades de los niños y niñas de 6 a 7 años. Espirales revistas multidisciplinaria de investigación Vol. 2 No. 15

Federación de Enseñanza de Andalucía. (2011). La estimulación temprana y su importancia. Temas para la Educación, 3-10.

Huamán, ML., (2018), Bases científicas que sustentan la estimulación temprana de los niños de educación inicial. Trabajo académico presentado para optar el Título Profesional de Segunda Especialidad en Educación Inicial. Tumbes- Perú.

Huepp, F. L. (2005), Estimulación temprana a niños de cero a dos años con factores de riesgo de retraso mental. (Tesis de doctoral). Santiago de Cuba: Instituto Superior Pedagógico "Frank País García".

Maqueira, G., (2020), Conferencia Ier Foro Intercontinental sobre Pedagogía para la Educación Virtual. Ponente Invitada. Tema Necesidades Educativas Especiales, integración, inclusión y diversidad: Nuevos desafíos desde la Educación Virtual. Julio 8 - 2020.Universidad de Huacho. Perú

Maqueira, G (2020), Conferencias Modulo La estimulación Temprana en Instituciones Inclusivas. Maestría en Educación Inclusiva y Atención a la Diversidad. Módulo: Cohorte V. Plataforma MEV.

Ordoñez, M \& Tinajero, A. (2012). Estimulación temprana inteligencia emocional y cognitiva. España: MMVII

Ramírez P, Patiño V, Gamboa E, (2014)- La educación temprana para niños y niñas desde nacimiento a los 3 años: Tres perspectivas de análisis. Revista Electrónica Educare. Educare vol.18 n.3 Heredia Sep. /Dec. 2014. On-line versión ISSN 1409-4258Print versión ISSN 1409-4258.

Real Patronato de Prevención y de Atención a personas con discapacidad (2000) "Libro Blanco de la Atención Temprana". Ministerio de Trabajo y Asuntos Sociales. Documentos. 55/2000. Madrid. 
Sailema, AA., Maqueira, G., Sailema, M., Sailema Ríos, AD., (2019), Estrategia metodológica, esquema corporal y actividad física adaptada: Una trilogía para la inclusión social. Revista: Ciencia Digital Vol. 3, n².5, p. (186-196), https://doi.org/10.33262/cienciadigital.v3i2.5.539

Sánchez, A. (2011), La Estimulación Temprana de 0 a 3 años. Editorial Palabra ISBN: 9788482395128 (p 272) Madrid.

Terré Orlando, (2012) ¿Qué es la estimulación temprana? http://www.orlandoterre.com/art1.htm

UNICEF, (2011).Ejercicios de Estimulación Temprana. (2011) http desarrolloinfantiltemprano.mex.

UNIR (2020) Estimulación Temprana ¿Qué es y cuáles son sus ventajas?, Revista estimulación temprana. (www.unir.net) Consultado en Noviembre del (2020)

Zapata B, \& Restrepo J, (2013). Aprendizajes relevantes para los niños y las niñas en la primera infancia. Revista Latinoamericana de Ciencias Sociales, Niñez y Juventud, 11 (1),pp.217-227.http://revistalatinoamericanaumanizales.cinde.org.co.

DOI:10.11600/1692715x.11114270712.

Zapata, B. \& Ceballos, L. (2010). Opinión sobre el rol y perfil del educador para la primera infancia. Revista Latinoamericana de Ciencias Sociales, Niñez y Juventud, 8 (2), pp. 1069-1082.

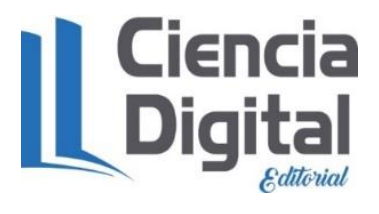


PARA CITAR EL ARTÍCULO INDEXADO.

Ríos Bayas, R. S., Coral Padilla, S. J., Carrasco Coca, O. R., \& Espinoza Regalado, C. E. (2021). La estimulación temprana como base para los procesos de enseñanza-aprendizaje en la educación infantil. Ciencia Digital, 5(1), 252-271. https://doi.org/10.33262/cienciadigital.v5i1.1543

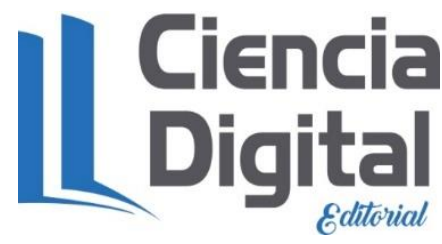

El artículo que se publica es de exclusiva responsabilidad de los autores y no necesariamente reflejan el pensamiento de la Revista Ciencia Digital.

El artículo queda en propiedad de la revista y, por tanto, su publicación parcial y/o total en otro medio tiene que ser autorizado por el director de la Revista Ciencia Digital.
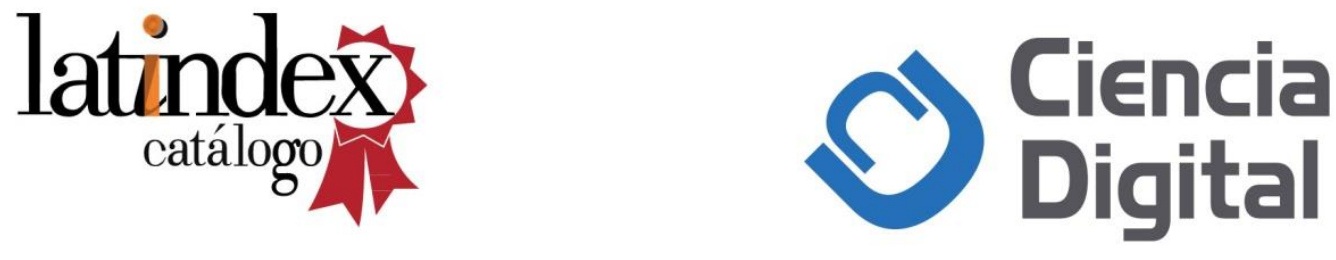